\title{
Reduction of Methylglyoxal-Induced Glycation by Pyridoxamine Improves Adipose Tissue Microvascular Lesions
}

\author{
Tiago Rodrigues, ${ }^{1}$ Paulo Matafome, ${ }^{1,2}$ Daniela Santos-Silva, ${ }^{1}$ \\ Cristina Sena, ${ }^{1}$ and Raquel Seiça ${ }^{1}$ \\ ${ }^{1}$ Laboratory of Physiology, Institute of Biomedical Research on Light and Image (IBILI), Faculty of Medicine, \\ University of Coimbra, 3000-354 Coimbra, Portugal \\ ${ }^{2}$ Center of Ophthalmology, IBILI, Faculty of Medicine, University of Coimbra, 3000-354 Coimbra, Portugal
}

Correspondence should be addressed to Paulo Matafome; paulomatafome@gmail.com

Received 14 January 2013; Revised 26 February 2013; Accepted 1 March 2013

Academic Editor: Adelino Leite Moreira

Copyright (C) 2013 Tiago Rodrigues et al. This is an open access article distributed under the Creative Commons Attribution License, which permits unrestricted use, distribution, and reproduction in any medium, provided the original work is properly cited.

Background and Aims. Adipose tissue dysfunction results from many factors, including glycation-induced microvascular damages. We tested the usefulness of inhibiting methylglyoxal-induced glycation to adipose tissue microvasculature in this work, using the antioxidant and dicarbonyl scavenger drug pyridoxamine. Methods. A group of Wistar rats was treated daily with methylglyoxal (MG, $75 \mathrm{mg} / \mathrm{Kg} /$ day, 8 weeks). Half of this group was treated with pyridoxamine in the following 4 weeks (Pyr) (100 mg/Kg/day) and the other half did not have any further treatment (MG). A group of Wistar rats without MG treatment was used as control (C). Results. MG group showed decreased HDL cholesterol and increased plasma free fatty acids levels, what was reverted by pyridoxamine. MG also caused an increase of tissue CEL levels (glycation marker), as well as increased staining of PAS and Masson Trichrome-positive components. Pyridoxamine led to CEL and TGF- $\beta$ levels similar to those observed in control rats and inhibited the accumulation of PAS and Masson Trichrome-positive components. MG caused a decrease of Bcl-2/Bax ratio (marker of apoptosis) and vWF staining (microvascular marker), what was partially reverted by the treatment with pyridoxamine. Conclusions. Preventing methylglyoxal-induced accumulation of glycated and fibrotic materials using pyridoxamine improves the microvascular lesions of the adipose tissue.

\section{Introduction}

Adipose tissue dysfunction relies on many and heterogeneous factors, including impaired secretory function, insulin resistance, and lipolysis, which are believed to be caused by the activation of inflammatory mechanisms. In turn, inflammation is activated as a consequence of excessive lipid uptake, adipocyte hypertrophy, and hypoxia. Besides the limited ability of oxygen to diffuse between hypertrophic adipocytes, hypoxia also results from microvascular dysfunction and decreased compensatory angiogenesis [14]. These mechanisms are thought to contribute to the development and progression of type 2 diabetes. Recently, our group demonstrated that microvascular dysfunction and concomitant hypoxia may be caused by methylglyoxalinduced glycation, what may constitute a new factor for adipose tissue dysfunction during type 2 diabetes progression [1]. Thus, new strategies improving microvascular function of adipose tissue may strongly contribute to prevent these mechanisms.

Methylglyoxal (MG) is a highly reactive dicarbonyl compound, endogenously formed during lipid peroxidation and glycolysis. Irreversible formation of advanced glycation endproducts (AGE) from methylglyoxal is one of the pathways responsible for diabetes-associated complications, not only due to increased aminoacid crosslinks and protein misfolding, but also due to the RAGE activation (AGE receptor) $[2,3]$. We showed that increased methylglyoxalinduced AGEs accumulation in adipose tissue of Wistar rats leads to hypoadiponectinemia, increased plasma free fatty acids, fibrosis, decreased irrigation, hypoxia, higher levels of apoptotic markers (ratio Bcl-2/Bax and caspase 3), and 
macrophage recruitment [1]. As well, other authors showed that MG-induced AGE accumulation leads to microvascular dysfunction in other models [5-8].

Pyridoxamine is a vitamin $\mathrm{B}_{6}$ derivative, which belongs to the $\mathrm{B}$ vitamins family, a group of vitamins known as coenzymes of several metabolic cellular pathways. Pyridoxamine is known to be an inhibitor of the Maillard reaction and a blocker of AGE formation from Amadori products, mainly because it acts as a dual scavenger of free radical and carbonyl species. Pyridoxamine was observed to decrease AGE accumulation, in part due to increased glyoxalase-I expression and decreased oxidative stress and RAGE levels in several models of diabetic complications [6, 9-12].

This work was designed to assess the usefulness of AGE inhibition in the adipose microvasculature, after methylglyoxal-induced glycation. Pyridoxamine was selected due to its double inhibitory functions over AGE formation.

\section{Materials and Methods}

2.1. Reagents. Unless otherwise stated all reagents were purchased to Merck Darmstadt (Germany), Sigma-Aldrich (EUA) or Pancreac Química SA (Spain). Antibodies used were directed to actin (MAB1501R, Millipore, USA), CEL (KH025, TransGenic Inc., Japan), RAGE (ab3611, Abcam, UK), TGF- $\beta$ (MAB240, R\&D Systems Inc.), Bax, Bcl-2 (SC 6236 and SC7382, Santa Cruz Biotechnology, USA), and vWF (A0082, DAKO, USA).

2.2. Animal Models. In this work we studied 6-month-old Wistar rats, from our breeding colonies at the Faculty of Medicine, University of Coimbra. A group of Wistar rats was treated with methylglyoxal in the daily water (Sigma, USA) during 8 weeks $(75 \mathrm{mg} / \mathrm{Kg} /$ day, diluted in the water). After this period, these rats were divided in two subgroups: one was treated with Pyridoxamine (Pyr) (Sigma, USA) during the following 4 weeks $(100 \mathrm{mg} / \mathrm{Kg} /$ day diluted in the daily water) $[13,14]$, whereas the other did not have any kind of treatment (MG). The control group (C) did not have any treatment during the whole period of the experiment ( $n=6$ per group). MG administration was suspended at the moment of pyridoxamine treatment initiation, in order to avoid ex vivo interactions with pyridoxamine. Animals were kept (2 per cage) under standard ventilation, temperature $\left(22-24^{\circ} \mathrm{C}\right)$, humidity (50-60\%), and light (12 hours light/12 hours darkness) with free access to water and food (standard diet AO4, Panlab, Barcelona). In treated animals, water consumption was monitored daily for dosage adjustment purposes. The experimental protocol was approved by the local Institutional Animal Care and Use Committee, and all the procedures were performed by licensed users (FELASA).

2.3. In Vivo Analysis and Sample Collection. At the end of the treatment, body weight was determined. Glycemia, fasting (16 hours) and 2 hours after intraperitoneal glucose administration $(1.8 \mathrm{~g} / \mathrm{Kg})$, was measured in the tail vein through the glucose oxidase method, using a glucometer and reactive test stripes (Elite-Bayer SA, Portugal). Glycated hemoglobin was determined using the DCA2000+ system (Siemens, Portugal).

Blood samples were obtained through cardiac puncture from anesthetized animals (ketamine chloride $(75 \mathrm{mg} / \mathrm{kg}$, i.m., Parke-Davis, Ann Arbor, USA) and chlorpromazine chloride $(2.65 \mathrm{mg} / \mathrm{kg}$, im, Lab. Vitória, Portugal)), after 16 hours fasting. Serum and plasma were collected using tubes BD Vacutainer and BD Vacutainer K3E, with 5.4 mg EDTA (UK), respectively. Blood was centrifuged at $3500 \times \mathrm{g}, 4^{\circ} \mathrm{C}, 10$ minutes and plasma and serum were aliquoted and stored at $-80^{\circ} \mathrm{C}$. After blood collection, animals were sacrificed and epididymal adipose tissue was harvested, washed in $0.9 \%$ $\mathrm{NaCl}$, and immediately stored in $10 \%$ formalin or frozen at $-80^{\circ} \mathrm{C}$.

Serum levels of cholesterol (total and HDL) and triglycerides were determined using commercial kits (OlympusDiagnóstica, Portugal, Produtos de Diagnóstico SA, Portugal). Serum concentration of adiponectin was determined by commercially available ELISA kits (Adiponectin immunoassay kit KRP0041, Invitrogen). Plasma levels of free fatty acids were assessed spectrophotometrically using the half-micro test (11383175001 Roche Diagnostic, Germany).

2.4. Western Blotting. Tissue sections of $300 \mathrm{mg}$ were homogenized in a buffer containing $25 \mathrm{mM}$ Tris, $150 \mathrm{mM} \mathrm{NaCl}$, 1\% Triton X-100, 1 mM EDTA, 1 mM EGTA, 10 mM PMSF, and $40 \mu \mathrm{L} / \mathrm{g}$ tissue of proteases inhibitor cocktail (Sigma, USA), $\mathrm{pH}=7.7$ and centrifuged at $14000 \times \mathrm{g}, 20$ minutes, $4^{\circ} \mathrm{C}$. Supernatants were collected, centrifuged again, and protein concentration was determined using the BCA method (Pierce, USA). Samples ( $50 \mu \mathrm{g}$ ) were separated by SDS-PAGE in $10 \%$ acrylamide gels and transferred to PVDF membranes. Membranes were blocked with TBST solution (25 mM Tris$\mathrm{HCl}, 150 \mathrm{mM} \mathrm{NaCl}, 0.1 \%$ Tween, $\mathrm{pH}=7.6$ ) supplemented with $5 \%$ BSA. Membranes were then incubated overnight at $4^{\circ} \mathrm{C}$ with the respective primary antibodies (CEL, RAGE, TGF- $\beta$, Bcl-2 and Bax, diluted $1: 1000$ in TBST solution supplemented with 1\% BSA) and during 2 hours at room temperature with the secondary antibodies (anti-rabbit/mouse 1:10000; GE Healthcare, UK). Membranes were incubated with ECF and revealed using the Typhoon system (GE Healthcare Life Sciences, UK). Membrane analysis was performed using the software Image Quant (Molecular Dynamics, USA).

2.5. Histology. Tissue sections $(4 \mu \mathrm{m})$ were stained with Periodic Acid Schiff (PAS; carbohydrates and glycoconjugates) and Masson Trichrome (collagen/connective tissue), two slices per animal, three animals per group. Immune staining was performed after paraffin removal, hydration, and blocking, following the recommendation of the manufacturer (DAB150 immunoperoxidase secondary detection system JH1743622, Millipore, USA). Sections were incubated overnight at $4^{\circ} \mathrm{C}$ with the primary antibody (TGF- $\beta$ and vWF, diluted 1:100 in PBS) and during 1 hour at room temperature with the secondary antibodies (Alexa Fluor 488 goat anti-rabbit IgG and Alexa Fluor 568 goat antimouse IgG, Invitrogen, USA). Finally, sections were mounted with mounting medium (DAKO, USA) and analyzed in 


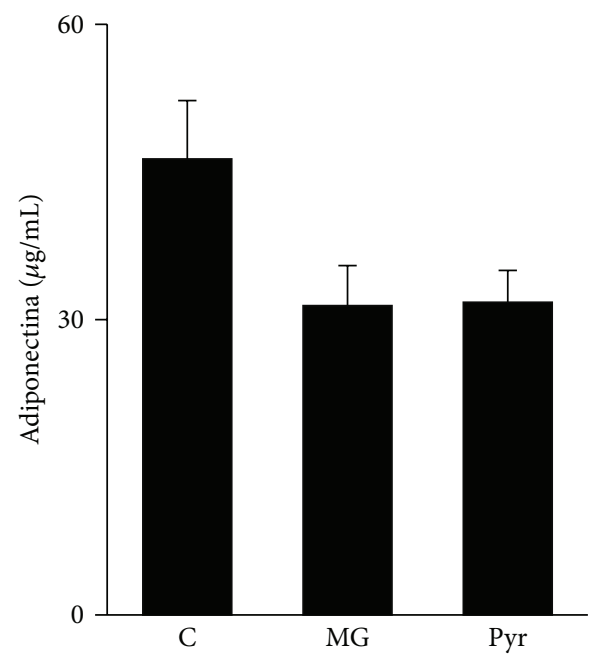

(a)

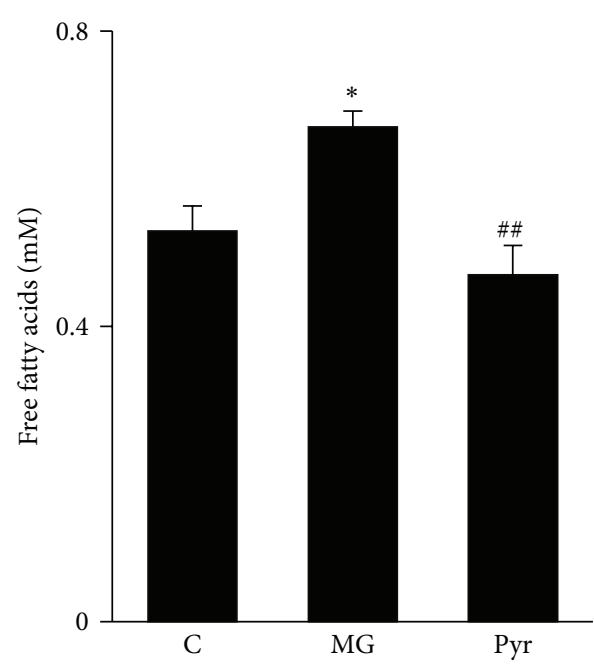

(b)

Figure 1: Serum adiponectin $(\mu \mathrm{g} / \mathrm{mL})$ (a) and plasma free fatty acids levels $(\mathrm{mM})$ (b). C: Control Wistar rats; MG: Wistar rats with methylglyoxal administration; Pyr: Wistar rats with methylglyoxal administration and pyridoxamine treatment. $n=6$ in each group. ${ }^{*}$ Different from C. ${ }^{\#}$ Different from MG. 1 symbol $P<0.05 ; 2$ symbols $P<0.01$.

a fluorescent microscope (Leica DMIRE2, Leica Microsystems, Germany). Fluorescence was adjusted to avoid background fluorescence of paraffin. For vWF quantification ten images were captured randomly (images containing large vessels were excluded) from two slices per animal/three animals per group. Fluorescence quantification was performed using the software Image (NIH, USA).

2.6. Statistical Analysis. Results are presented as mean \pm SEM. One-way ANOVA test was used to determine statistical differences. Post hoc Tukey test was performed to assess the differences between groups. $P<0.05$ was considered significant.

\section{Results}

3.1. Metabolic Profile and Functional Systemic Markers. MG administration during 8 weeks (MG group), as well as the treatment with pyridoxamine during 4 weeks (Pyr group), did not result in significant alterations of body weight, glycemia, HbAlc, triglycerides, and total cholesterol, compared to the control (C) group (Table 1). However, MG group showed a significant decrease of HDL cholesterol levels, compared to the $\mathrm{C}$ group $(P<0.05)$, which was reverted by pyridoxamine treatment (Pyr group) $(P<0.05)$ (Table 1). Regarding serum adiponectin levels, no significant alterations were found (Figure 1(a)). On the other hand, MG administration led to increased circulating free fatty acids levels, when compared to $C$ group $(P<0.05)$, suggesting adipocyte dysfunction. In turn, the treatment with pyridoxamine normalized plasma free fatty acids levels $(P<$ 0.01) (Figure 1(b)).
TABLE 1: Body weight, glycemia, fasting and 2 hours after intraperitoneal glucose injection $(1.8 \mathrm{~g} / \mathrm{kg})$, glycated hemoglobin, serum triglycerides, and total and HDL cholesterol.

\begin{tabular}{lccc}
\hline Parameter & C & MG & Pyr \\
\hline Body weight $(\mathrm{g})$ & $430 \pm 17$ & $440 \pm 18$ & $433 \pm 12$ \\
Fasting glycemia (mg/dL) & $63.3 \pm 1$ & $62.9 \pm 1.5$ & $64.3 \pm 2$ \\
Glycemia at 2 h (mg/dL) & $92.0 \pm 6.1$ & $91.3 \pm 1.8$ & $92.5 \pm 3.1$ \\
HbAlc (\%) & $3.6 \pm 0.1$ & $3.6 \pm 0.0$ & $3.7 \pm 0.1$ \\
Triglycerides (mg/dL) & $95.8 \pm 9.1$ & $78.6 \pm 5.6$ & $98 \pm 10.8$ \\
Total cholesterol (mg/dL) & $92.5 \pm 5.8$ & $84.7 \pm 4.7$ & $91.3 \pm 4.5$ \\
HDL cholesterol (mg/dL) & $55.5 \pm 1.2$ & $47.3 \pm 2.0^{*}$ & $54.8 \pm 1.6^{\#}$ \\
\hline
\end{tabular}

C: Control Wistar rats; MG: Wistar rats with methylglyoxal administration; Pyr: Wistar rats with methylglyoxal administration and pyridoxamine treatment. Data is presented as mean $\pm \mathrm{SEM}, n=6$ in each group. ${ }^{*}$ Different from C; ${ }^{\#}$ different from MG. 1 symbol $P<0.05$.

\subsection{Tissue Markers of Microvascular Dysfunction}

3.2.1. Glycation. Glycation is known to be related to microvascular dysfunction. MG treatment during 8 weeks resulted in increased $\mathrm{N}$-epsilon-(carboxyethyl)lysine (CEL) accumulation in adipose tissue, compared to the $\mathrm{C}$ group $(P<0.01)$ (Figure 2(a)), but did not cause significant alterations of RAGE expression (Figure 2(b)). Pyridoxamine administration significantly reverted CEL accumulation in adipose tissue $(P<0.01)$, leading to values similar to control rats (Figure 2(a)). PAS staining represents the accumulation of carbohydrates and nonspecific glycated materials. In accordance with CEL levels, a substantial increase of PAS material was observed in the adipose tissue of MG-treated rats, when compared to the $\mathrm{C}$ group. This accumulation was reverted by pyridoxamine treatment, leading to a phenotype similar to control rats (Figure 3(a)). 

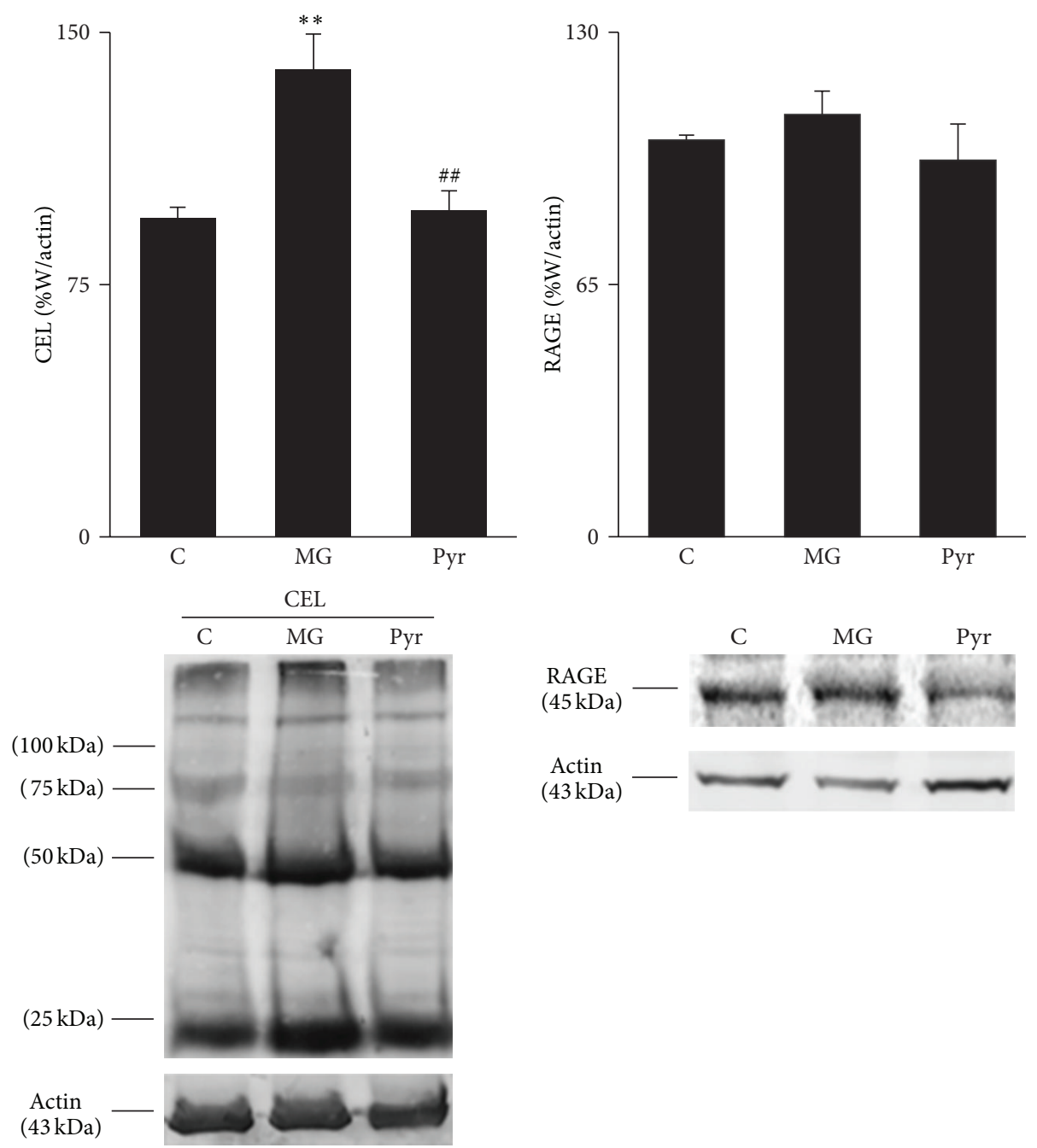

(a)

(b)

FIGURE 2: Adipose tissue levels of CEL (a) and RAGE (b), calculated as percentage of C. Representative Western blots of CEL and RAGE are shown. C: Control Wistar rats; MG: Wistar rats with methylglyoxal administration; Pyr: Wistar rats with methylglyoxal administration and pyridoxamine treatment. $n=6$ in each group. ${ }^{*}$ Different from C. ${ }^{\#}$ Different from MG. 2 symbols $P<0.01$.

3.2.2. Fibrosis. Masson Trichrome staining shows components rich in collagen. The administration of MG during 8 weeks resulted in more fibrotic components in the adipose tissue. More, the staining was observed in PASpositive regions. Once again, the treatment with pyridoxamine resulted in decreased fibrotic material, resulting in a phenotype similar to control rats (Figure 3(b)). As well, the transforming growth factor- $\beta$ (TGF- $\beta$ ) precursor was observed to be decreased after pyridoxamine treatment, when compared to the MG group $(P<0.05)$ (Figure $4(\mathrm{a}))$. No significant differences were observed in the cleaved form of the TGF- $\beta$ (Figure 4(b)). The histological staining of TGF- $\beta$ showed accumulation in the capillaries, as suggested by its colocalization with the Von Willebrand factor (Figure 4(c)).

3.2.3. Apoptosis. The ratio between $\mathrm{Bcl}-2$ and Bax can be used as an indicator of the predisposition for cellular death by apoptosis. The decrease of this ratio in the MG group, when compared to the $\mathrm{C}$ group $(P<0.01)$, indicates that MGinduced glycation leads to a cellular death predisposition. These effects were completely reverted by the treatment with pyridoxamine $(P<0.01)$ (Figure $5(\mathrm{a}))$.

3.2.4. Endothelial Dysfunction. Quantification of vWF fluorescent staining was used as a marker of adipose tissue microvasculature, as it is a marker of endothelial cells and is present in the circulation. MG administration resulted in a significant decrease of adipose tissue vWF levels, when compared to control rats $(P<0.05)$. After pyridoxamine treatment a significant difference in relation to the $C$ group was no longer observed, despite that no significant differences were observed between MG and Pyr groups (Figure 5(b)).

\section{Discussion}

This study investigates the scavenger properties and beneficial actions of pyridoxamine in methylglyoxal-induced 

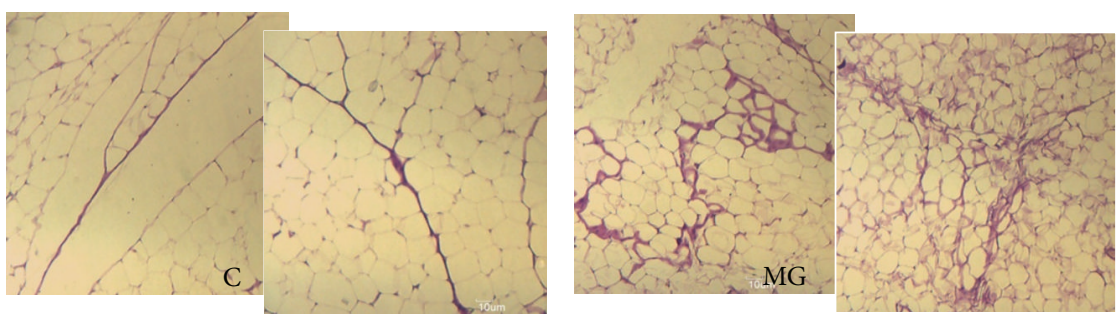

(a) PAS (200X)
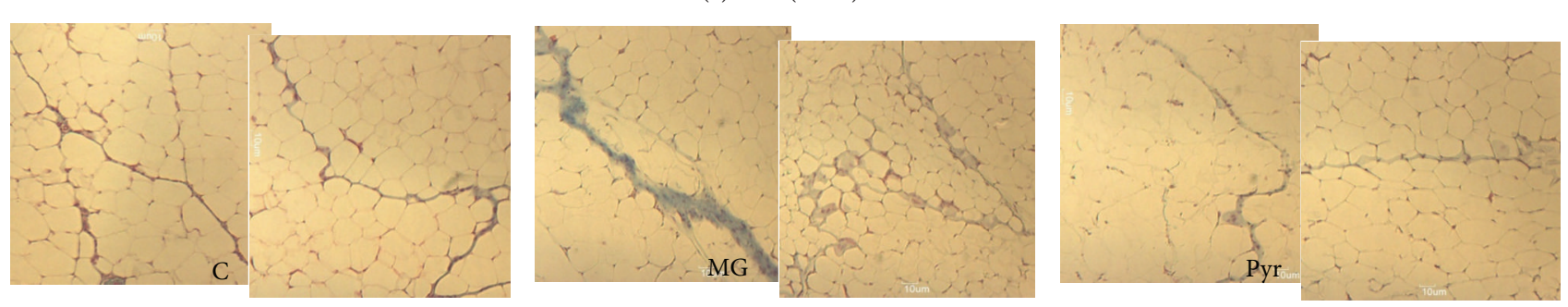

(b) Masson Trichrome (200X)

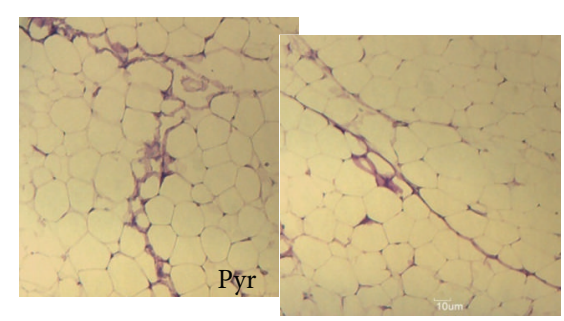
FIGURE 3: Histological analysis of adipose tissue show PAS staining (200X) (a) and Masson Trichrome staining (200X) (b). C: Control Wistar
rats; MG: Wistar rats with methylglyoxal administration; Pyr: Wistar rats with methylglyoxal administration and pyridoxamine treatment.

microvascular damages in the adipose tissue. The significant alterations of HDL cholesterol, free fatty acids, CEL, PAS staining, TGF- $\beta$ precursor, Masson Trichrome staining, Bcl$2 /$ Bax ratio, and partially vWF were reverted by pyridoxamine. In accordance with previous reports from our laboratory, MG administration during 8 weeks did not have significant alterations in body weight, glycemia, and HbAlc [1, 7]. Once no alterations were found in the glucose metabolism, the lesions observed after MG treatment are only due to direct MG effects. However, MG administration increased systemic free fatty acids levels, suggesting adipose tissue dysfunction, due to a higher rate of lipolysis. These results are also in accordance with our previous observations after MG treatment during 14 weeks [1]. As demonstrated before in Zucker diabetic rats (ZDF) $[13,15]$, pyridoxamine effects led to lower systemic levels of free fatty acids. Our previous and present results show that MG treatment leads to decreased serum adiponectin levels, but these effects may be acquired during the time, as they are significant only after 14 weeks of MG administration [1, 7]. MG treatment during 8 weeks may have a lower impact on adiponectin levels, or the effects may have been lost because the rats were kept with water during 4 weeks after MG treatment.

CEL was determined as it is an important AGE formed directly from the MG [8]. Previously, correlation between CEL levels and MG administration was observed in the aorta of spontaneously hypertensive rats [16] and in retina of GLO1 transgenic rats [6]. As expected, MG administration during 8 weeks led to increased CEL levels and accumulation of PAS-positive material in adipose tissue, in accordance to previous reports $[1,17]$. However, 8 weeks of MG treatment were not sufficient to induce major alterations in RAGE levels in adipose tissue, what is in accordance with previous data from our laboratory (unpublished data). However, that kind of effect was observed in aorta after 14 weeks of MG administration [7].

Previous in vitro studies from Onorato and colleagues showed that pyridoxamine inhibits AGEs formation as well as subsequent protein modifications, features commonly observed in type 2 diabetes [18]. Several authors described that pyridoxamine inhibits AGE formation through the reaction with and scavenging of dicarbonyl intermediates [11, 19]. The role of pyridoxamine in decreasing AGE formation in in vitro systems [18] and in different animal models, including STZ-induced diabetic rats and ZDF rats, was also observed [11]. In accordance, in our study the treatment with pyridoxamine during 4 weeks after MG administration caused a significant decrease of CEL levels and PAS-positive components in adipose tissue.

Previous studies showed that MG induces microvascular damages and diabetes-like complications. In vivo studies using Wistar rats showed an increase of TGF- $\beta$ in glomerular, tubular, interstitial, and endothelial cells of the kidney [17]. In vitro, collagen glycation caused by MG stimulates the differentiation of human cardiac fibroblasts to myofibroblast, in a TGF- $\beta$-dependent manner. This process was described as critical for the development of fibrosis in diabetes [20]. As well, we reported that MG administration during 14 weeks also led to increased TGF- $\beta$ expression, as well as increased Masson Trichrome staining [1]. In the present study, we show that even after 8 weeks of MG treatment a higher accumulation of Masson Trichrome components occurred. In vitro studies with pyridoxamine revealed that it protects from MG-induced integrin binding and cell adhesion, a major event of vessel thickening [10]. Here, we show that the treatment with pyridoxamine decreased the expression of TGF- $\beta$ precursor and inhibited the accumulation of Masson Trichrome-positive material, suggesting a causal role between 

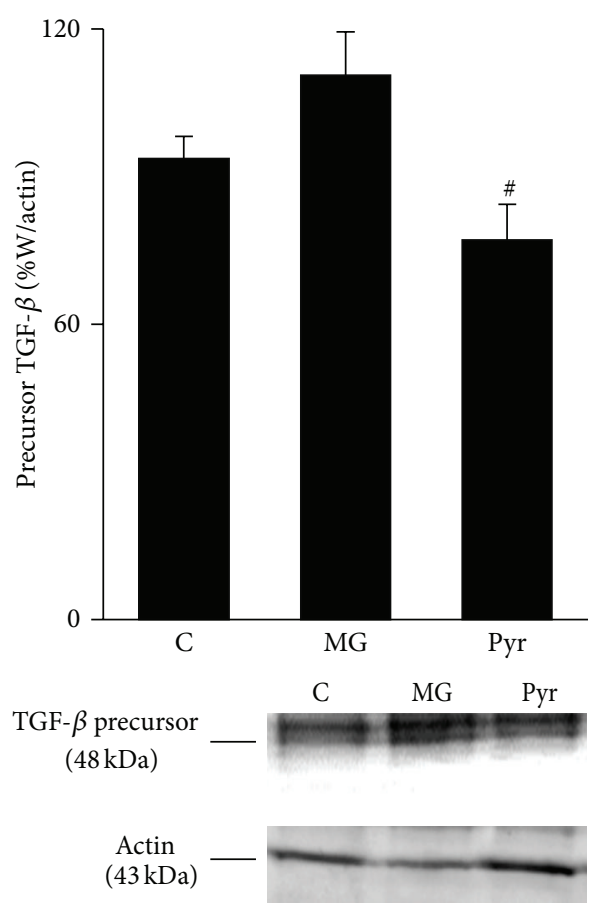

(a)

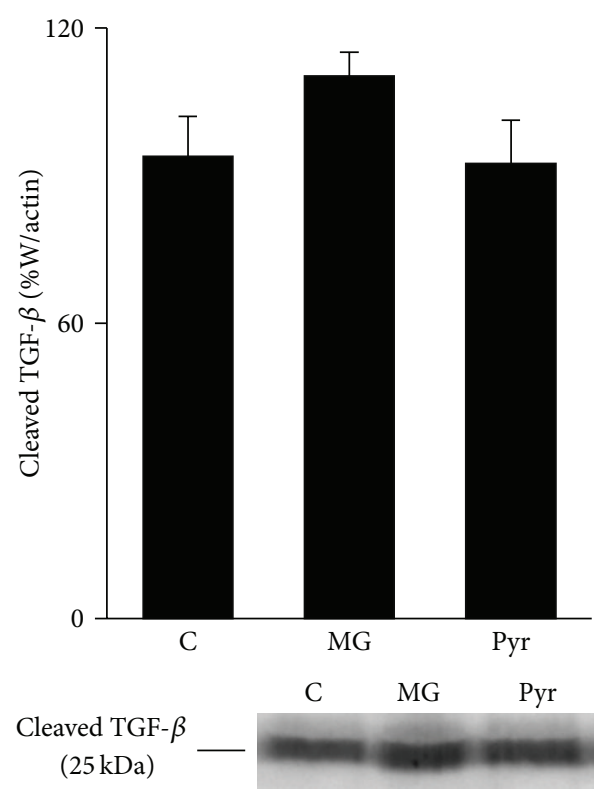

(b)
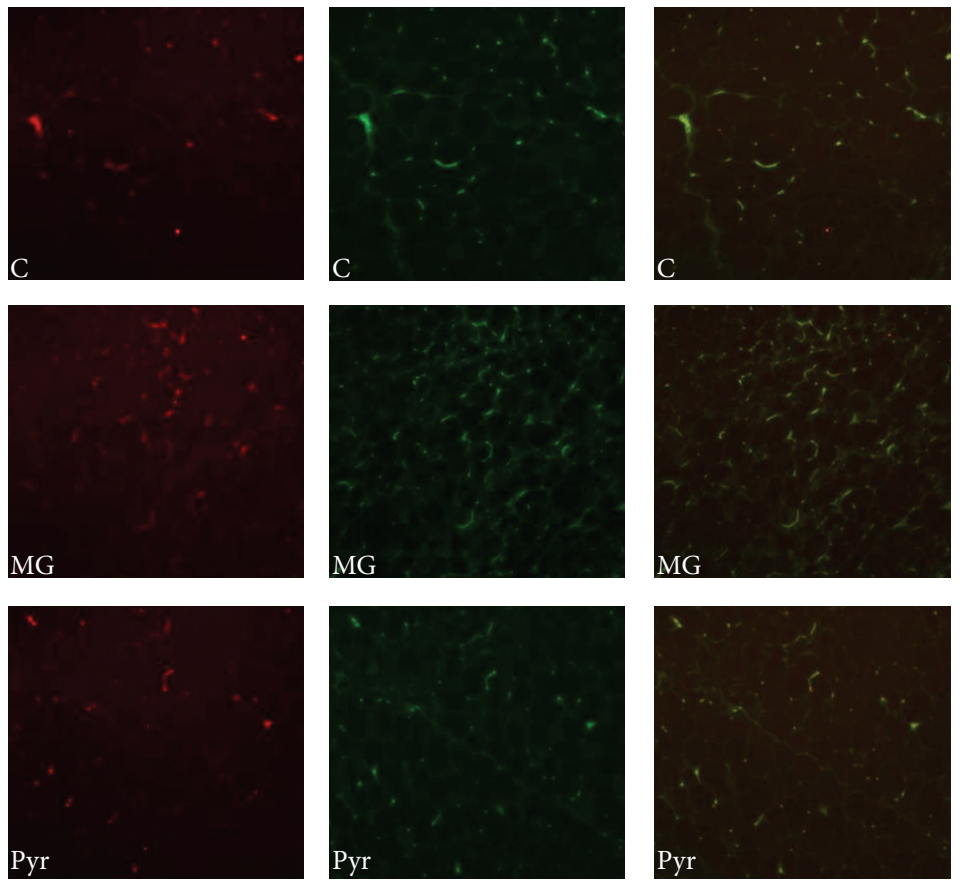

(c) TGF $\beta$ (red) and vWF (green) (200X)

FIGURE 4: Adipose tissue levels of precursor (a) and cleaved (b) forms of TGF- $\beta$, calculated as percentage of W. Representative Western blots of TGF- $\beta$ forms are shown. Immunohistochemistry images of adipose tissue show TGF- $\beta$ (red), vWF (green), and merge staining (200X) (c). C: Control Wistar rats; MG: Wistar rats with methylglyoxal administration; Pyr: Wistar rats with methylglyoxal administration and pyridoxamine treatment. $n=6$ in each group. ${ }^{\#}$ Different from MG. 1 symbol $P<0.05$.

increased glycation markers and profibrotic responses. Colocalization of TGF- $\beta$ and vWF suggests TGF- $\beta$ expression to occur mostly in blood vessels regions. This demonstrates the presence of fibrosis in the microvasculature of adipose tissue, what may be an important factor for local microvascular lesions.

The ratio between $\mathrm{Bcl}-2$ and $\mathrm{Bax}$ decreased after MG treatment, what is in accordance with several reports from 


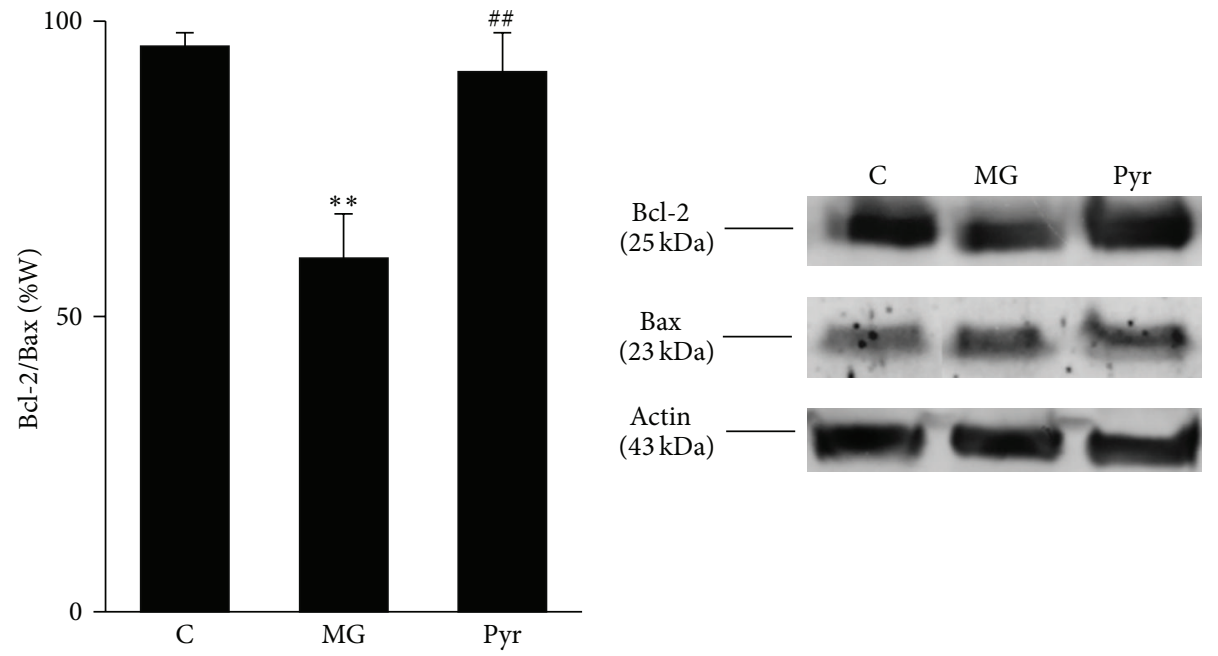

(a)

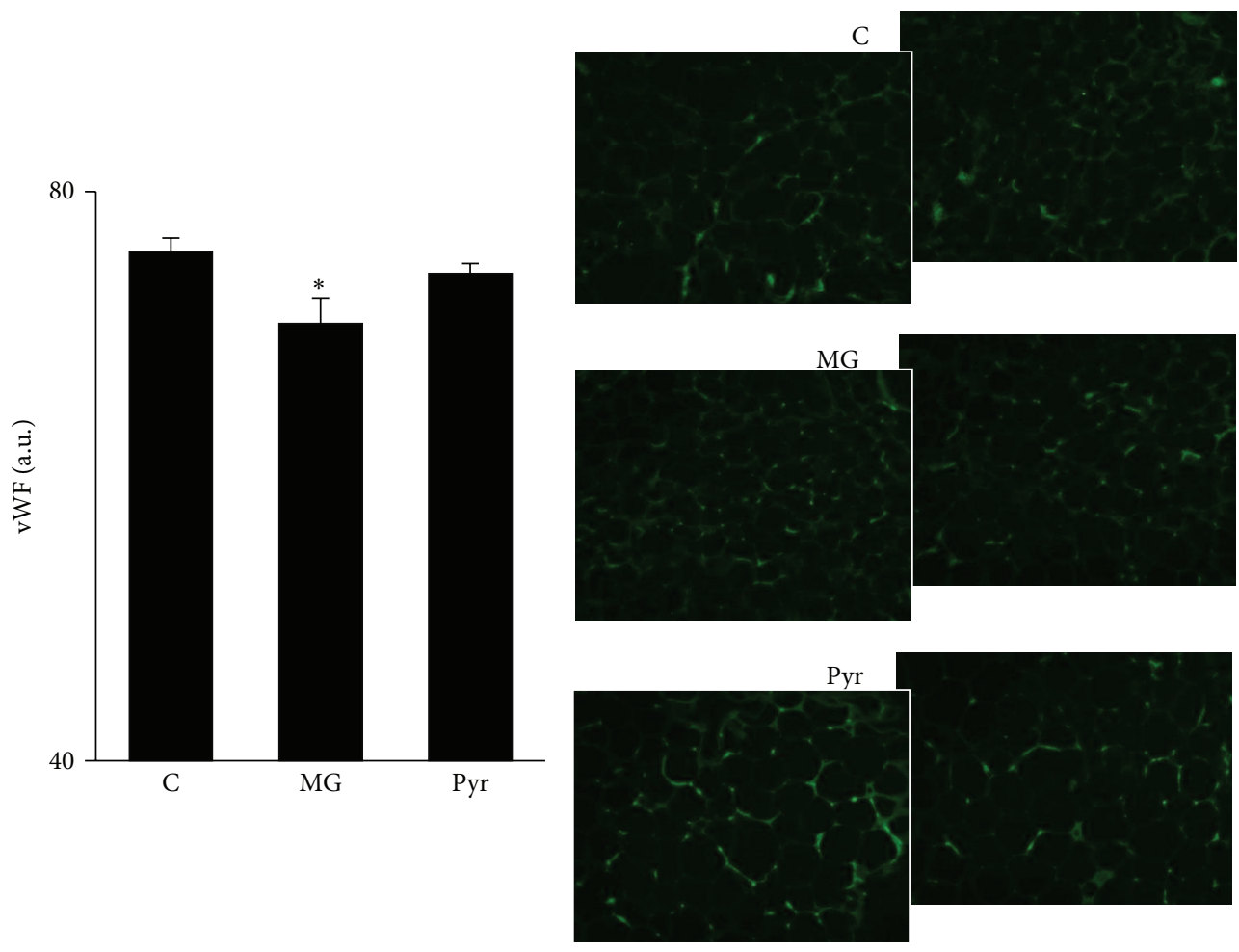

(b)

FIGURE 5: Bcl-2/Bax ratio in adipose tissue: (a) representative Western blots of Bcl-2 and Bax are shown. Immunohistochemistry images show vWF staining, and the respective quantification of fluorescence (b). C: Control Wistar rats; MG: Wistar rats with methylglyoxal administration; Pyr: Wistar rats with methylglyoxal administration and pyridoxamine treatment. $n=6$ in each group. ${ }^{*}$ Different from C. ${ }^{\#}$ Different from MG. 1 symbol $P<0.05 ; 2$ symbols $P<0.01$.

our and other laboratories [1,21,22]. Our group demonstrated that decreased $\mathrm{Bcl}-2 / \mathrm{Bax}$ ratio is associated with increased endothelial cell death and higher vessel dysfunction in the retina [21]. Furthermore, we demonstrated that this event is also related with decreased blood supply to adipose tissue, what may lead to hypoxia, a major contributor to adipocyte dysfunction [1]. Pyridoxamine was observed to prevent cell death by apoptosis in the human lens epithelial cell line HLE-B3 and in the cataractous lenses of ZDF rats, through the inhibition of argpyrimidine formation $[5,23]$. In $\mathrm{B} 6 \mathrm{db} / \mathrm{db}$ mice, a model of type 2 diabetes, pyridoxamine decreased the progression of established diabetic nephropathy [14].

As mentioned, we previously reported that MG administration during 14 weeks causes a decrease of blood supply to the adipose tissue, mainly through increased endothelial 
cell death and impaired angiogenesis. In this study, we show that these effects were similar after 8 weeks, although more modest. Despite pyridoxamine was effective in the reduction of most of the lesion markers, it was not able to significantly raise adipose vWF levels in relation to MG-treated rats. However, vWF levels were no longer decreased in relation to control rats, suggesting a partial effect.

\section{Conclusions}

We show that reducing adipose tissue glycation and fibrosis using pyridoxamine may be a useful strategy to improve adipose tissue microvascular lesions. This may contribute to prevent adipose tissue dysfunction during type 2 diabetes progression.

\section{Abbreviations}

W: Wistar rats

AGEs: Advanced glycation end products

CEL: N-epsilon-(carboxyethyl)lysine

MG: Methylglyoxal

PAS: $\quad$ Periodic acid of Schiff

RAGE: Advanced glycation end-product receptor

TGF: Transforming growth factor

vWF: Von Willebrand factor.

\section{Conflict of Interests}

The authors declare no conflict of interests.

\section{Acknowledgments}

The authors thank Serviço de Anatomia Patológica, Coimbra University Hospital, especially Ilda Simões for the support with the histological techniques. They thank Mário Simões from their laboratory for his technical support. The authors thank the Portuguese Foundation for Science and Technology (FCT, Project PTDC/SAU-OSM/67498/2006) and Faculty of Medicine, University of Coimbra.

\section{References}

[1] P. Matafome, D. Santos-Silva, J. Crisóstomo et al., "Methylglyoxal causes structural and functional alterations in adipose tissue independently of obesity," Archives of Physiology and Biochemistry, vol. 118, pp. 58-68, 2012.

[2] A. Goldin, J. A. Beckman, A. M. Schmidt, and M. A. Creager, "Advanced glycation end products: sparking the development of diabetic vascular injury," Circulation, vol. 114, no. 6, pp. 597605, 2006.

[3] A. Negre-Salvayre, R. Salvayre, N. Augé, R. Pamplona, and M. Portero-Otín, "Hyperglycemia and glycation in diabetic complications," Antioxidants and Redox Signaling, vol. 11, no. 12, pp. 3071-3109, 2009.

[4] G. H. Goossens, "The role of adipose tissue dysfunction in the pathogenesis of obesity-related insulin resistance," Physiology and Behavior, vol. 94, no. 2, pp. 206-218, 2008.
[5] J. Kim, O. S. Kim, C.-S. Kim, E. Sohn, K. Jo, and J. S. Kim, "Accumulation of argpyrimidine, a methylglyoxal-derived advanced glycation end product, increases apoptosis of lens epithelial cells both in vitro and in vivo," Experimental \& Molecular Medicine, vol. 44, pp. 167-175, 2012.

[6] A. K. Berner, O. Brouwers, R. Pringle et al., "Protection against methylglyoxal-derived AGEs by regulation of glyoxalase 1 prevents retinal neuroglial and vasodegenerative pathology," Diabetologia, vol. 55, pp. 845-854, 2012.

[7] C. Sena, P. Matafome, J. Crisastomo et al., "Methylglyoxal promotes oxidative stress and endothelial dysfunction," Pharmacological Research, vol. 65, pp. 497-506, 2012.

[8] A. V. Cantero, M. Portero-Otín, V. Ayala et al., "Methylglyoxal induces advanced glycation end product (AGEs) formation and dysfunction of PDGF receptor- $\beta$ : implications for diabetic atherosclerosis," FASEB Journal, vol. 21, no. 12, pp. 3096-3106, 2007.

[9] P. A. Voziyan and B. G. Hudson, "Pyridoxamine as a multifunctional pharmaceutical: targeting pathogenic glycation and oxidative damage," Cellular and Molecular Life Sciences, vol. 62, no. 15, pp. 1671-1681, 2005.

[10] V. K. Pedchenko, S. V. Chetyrkin, P. Chuang et al., "Mechanism of perturbation of integrin-mediated cell-matrix interactions by reactive carbonyl compounds and its implication for pathogenesis of diabetic nephropathy," Diabetes, vol. 54, no. 10, pp. 29522960, 2005.

[11] T. O. Metz, N. L. Alderson, S. R. Thorpe, and J. W. Baynes, "Pyridoxamine, an inhibitor of advanced glycation and lipoxidation reactions: a novel therapy for treatment of diabetic complications," Archives of Biochemistry and Biophysics, vol. 419, no. 1, pp. 41-49, 2003.

[12] F. Depeint, W. R. Bruce, N. Shangari, R. Mehta, and P. J. O'Brien, "Mitochondrial function and toxicity: role of B vitamins on the one-carbon transfer pathways," Chemico-Biological Interactions, vol. 163, no. 1-2, pp. 113-132, 2006.

[13] E. A. Muellenbach, C. J. Diehl, M. K. Teachey et al., "Interactions of the advanced glycation end product inhibitor pyridoxamine and the antioxidant $\alpha$-lipoic acid on insulin resistance in the obese Zucker rat," Metabolism, vol. 57, no. 10, pp. 1465-1472, 2008.

[14] F. Zheng, Y. J. Zeng, A. R. Plati et al., "Combined AGE inhibition and ACEi decreases the progression of established diabetic nephropathy in B6 db/db mice," Kidney International, vol. 70, no. 3, pp. 507-514, 2006.

[15] E. M. Muellenbach, C. J. Diehl, M. K. Teachey et al., "Metabolic interactions of AGE inhibitor pyridoxamine and antioxidant $\alpha$ lipoic acid following 22 weeks of treatment in obese Zucker rats," Life Sciences, vol. 84, no. 15-16, pp. 563-568, 2009.

[16] X. Wang, K. Desai, T. Chang, and L. Wu, "Vascular methylglyoxal metabolism and the development of hypertension," Journal of Hypertension, vol. 23, no. 8, pp. 1565-1573, 2005.

[17] J. Berlanga, D. Cibrian, I. Guillén et al., "Methylglyoxal administration induces diabetes-like microvascular changes and perturbs the healing process of cutaneous wounds," Clinical Science, vol. 109, no. 1, pp. 83-95, 2005.

[18] J. M. Onorato, A. J. Jenkins, S. R. Thorpe, and J. W. Baynes, "Pyridoxamine, an inhibitor of advanced glycation reactions, inhibits advanced lipoxidation reactions: mechanism of action of pyridoxamine," Journal of Biological Chemistry, vol. 275, no. 28, pp. 21177-21184, 2000. 
[19] R. G. Khalifah, Y. Chen, and J. J. Wassenberg, "Post-Amadori AGE inhibition as a therapeutic target for diabetic complications: a rational approach to second-generation Amadorin design," Annals of the New York Academy of Sciences, vol. 1043, pp. 793-806, 2005.

[20] A. Yuen, C. Laschinger, I. Talior et al., "Methylglyoxal-modified collagen promotes myofibroblast differentiation," Matrix Biology, vol. 29, no. 6, pp. 537-548, 2010.

[21] C. F. Bento, R. Fernandes, P. Matafome, C. Sena, R. Seia, and P. Pereira, "Methylglyoxal-induced imbalance in the ratio of vascular endothelial growth factor to angiopoietin 2 secreted by retinal pigment epithelial cells leads to endothelial dysfunction," Experimental Physiology, vol. 95, no. 9, pp. 955-970, 2010.

[22] W. H. Chan, H. J. Wu, and N. H. Shiao, "Apoptotic signaling in methylglyoxal-treated human osteoblasts involves oxidative stress, c-Jun N-terminal kinase, caspase-3, and p21-activated kinase 2," Journal of Cellular Biochemistry, vol. 100, no. 4, pp. 1056-1069, 2007.

[23] J. Kim, N. H. Kim, E. Sohn, C. S. Kim, and J. S. Kim, "Methylglyoxal induces cellular damage by increasing argpyrimidine accumulation and oxidative DNA damage in human lens epithelial cells," Biochemical and Biophysical Research Communications, vol. 391, no. 1, pp. 346-351, 2010. 


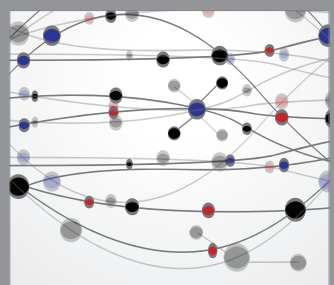

The Scientific World Journal
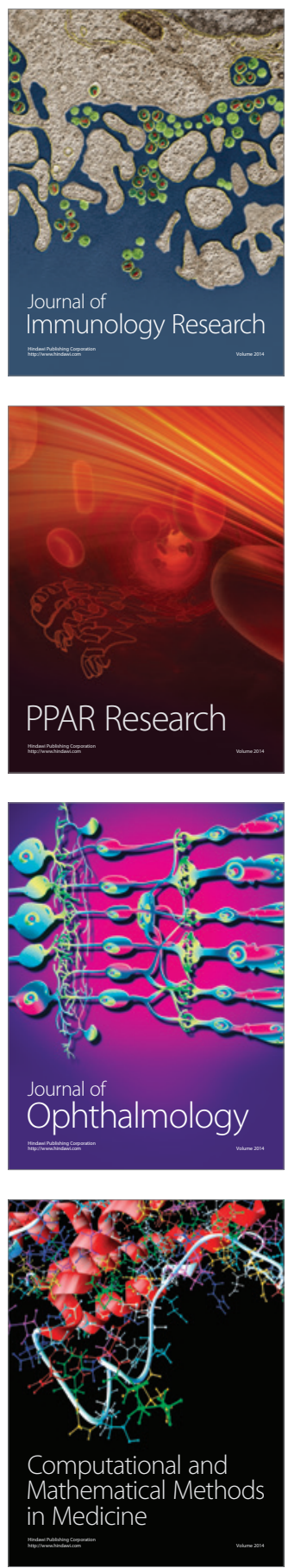

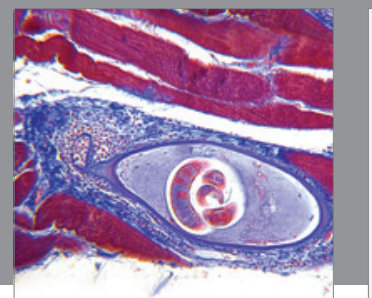

Gastroenterology

Research and Practice
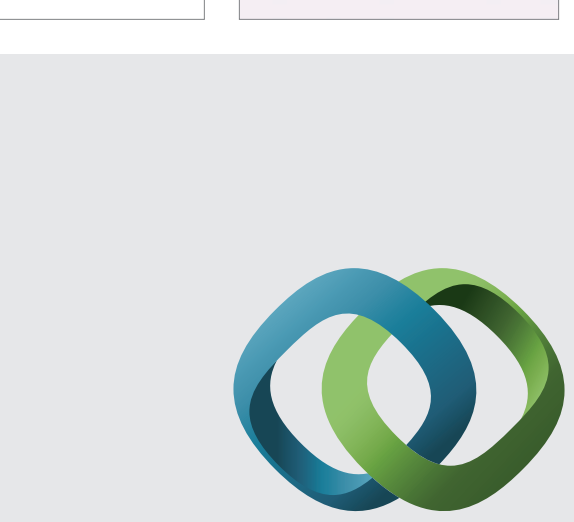

\section{Hindawi}

Submit your manuscripts at

http://www.hindawi.com
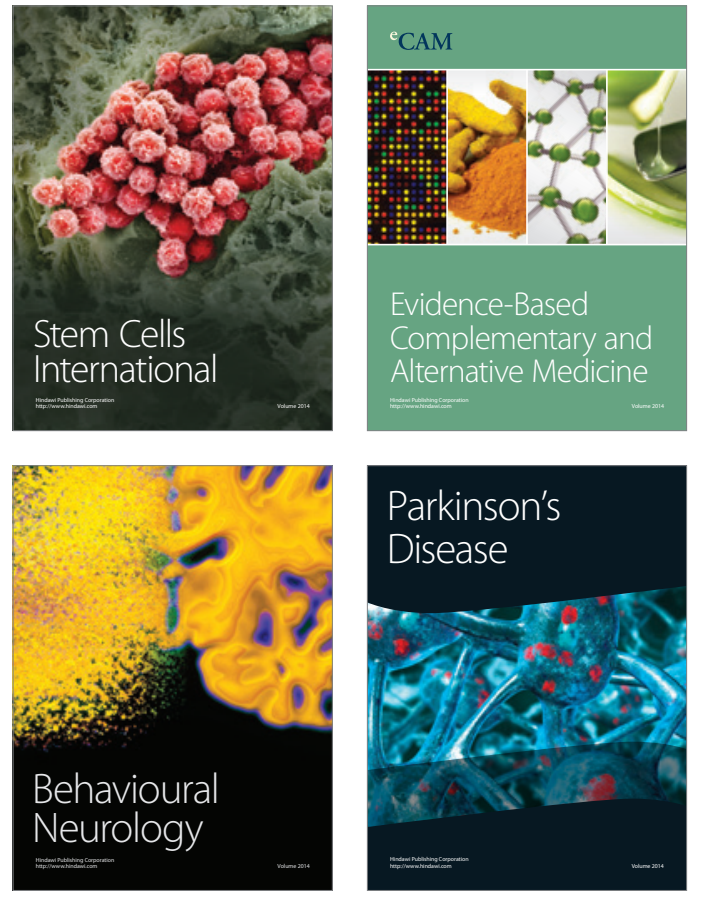
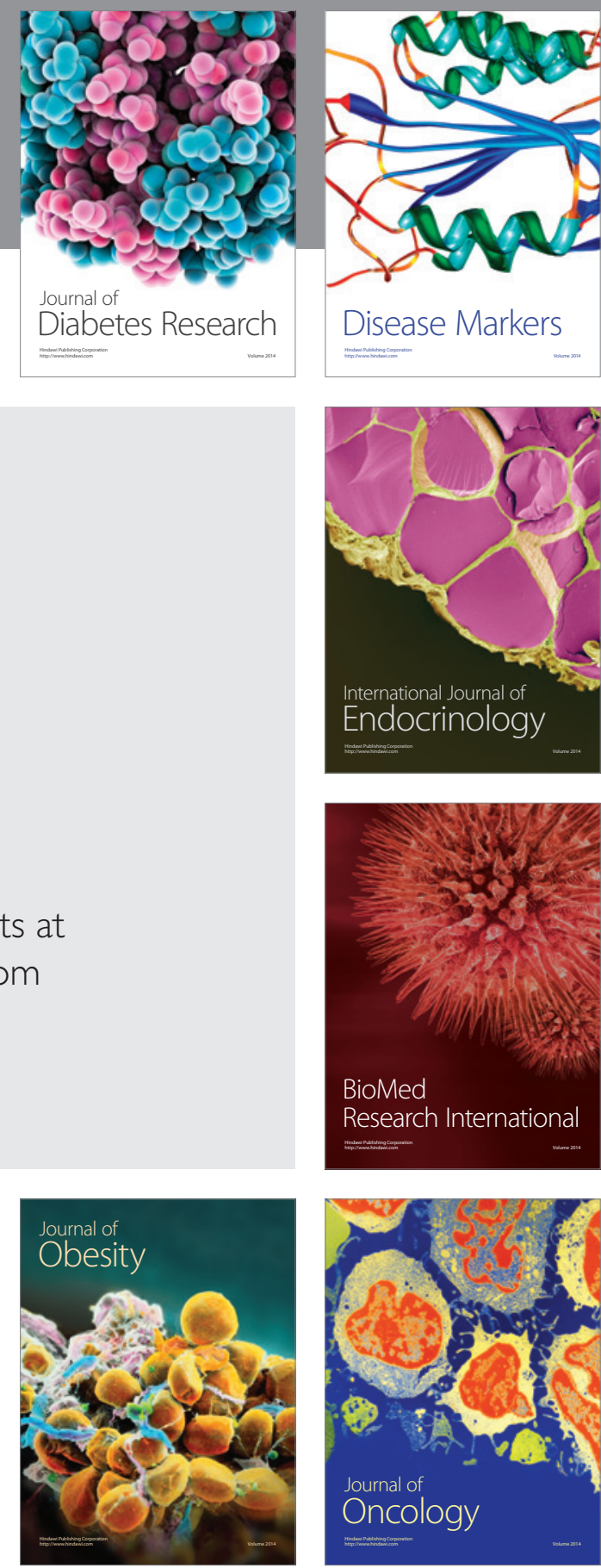

Disease Markers
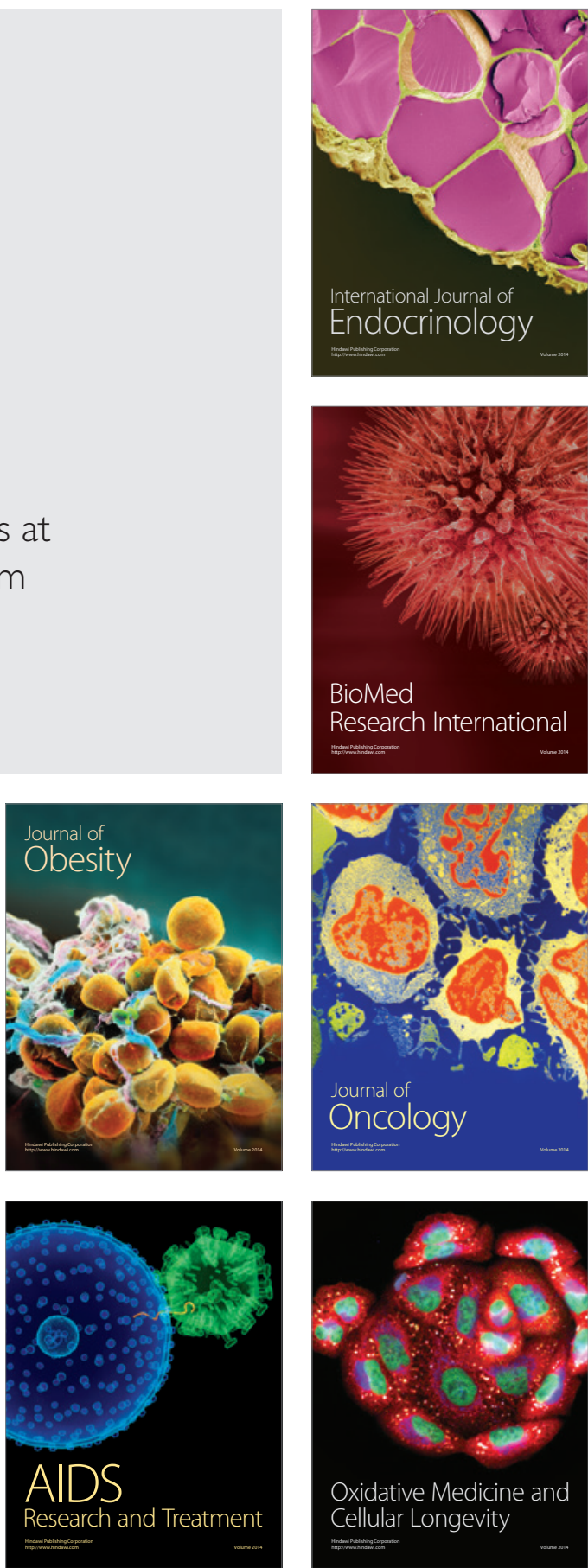